\title{
EXPRESSION DE LA VARIABILITÉ GÉNÉTIQUE ENTRE ÉTABLES DANS L'APTITUDE GÉNÉRALE DES MALES DE RACE A VIANDE AU CROISEMENT DE PREMIÈRE GÉNÉRATION
}

J.-C. Mocquot. - Station de Génétique quantitative et appliquée, C.N.R.Z., 78-Jouy-en-Josas.

Le choix traditionnel des reproducteurs bovins mâles est basé, en grande partie, sur la réputation de l'étable où ils sont nés. Ceci est, en particulier, le cas des taureaux destinés à engendrer des veaux de boucherie par croisement de première génération (la plupart du temps en dehors de la zone de sélection proprement dite de leur race). On peut apprécier objectivement l'intérêt d'une sélection de ce type, d'après la valeur estimée de la part de la variance génétique liée à des différences entre étables d'origine. Les méthodes de Robertson et Rendel, 1954 (J. Agric. Sci., 44, 184-192) et de M. Gilliand, 1952 ( $P h . D$. Thesis Ames, Iowa, U.S.A.) ne pouvaient être appliquées, puisqu'elles supposent que les descendants d'un reproducteur mâle sont répartis dans plusieurs étables et contrôlés en même temps que des animaux contemporains, cette dernière condition n'étant pas réalisée pour notre échantillon de calcul.

La méthode proposée consiste à utiliser les valeurs d'index de descendance calculées par ailleurs et à procéder à une décomposition de la variance de ses index "entre " et "intra "étable d'origine des reproducteurs mâles, en admettant les hypothèses classiques pour le modèle mathématique de représentation de l'index (en particulier action additive et indépendance des facteurs génétiques et du milieu). En supposant connus les effectifs de descendants par reproducteur et le coefficient d'héritabilité du caractère étudié, on peut ainsi estimer la part de la variance génétique liée à l'étable d'origine par rapport à la variance génétique totale.

Une application a été faite sur les index de poids à $2-3$ mois pour des taureaux de race limoulsine utilisés en insémination artificielle. Compte tenu d'une valeur d'héritabilité de 0,16 , on a estimé que l'étable d'origine expliquait 20 p. 100 de la variance génétique, résultat qui demande à être confirmé sur un plus grand nombre de données.

Phénomènes D'INTERACTION GÉNOTYPE $\times$ MILIEU DANS L'ÉlEVAGE DES Bovins A VIANDE EN ZONES DE MONTAGNE.

B. Vissac. - Station de Génétique quantitative et appliquée, C.N.R.Z., 78-Jouy-en-Josas.

C. BERANGer. - C.R.Z.V., 63-Theix par St-Genès-Champanelle.

Les montagnes d'Aubrac constituent une zone de pâturages d'altitude (800-1 $300 \mathrm{~m})$ où vit un cheptel autochtone exploité suivant le système ancien dans lequel le veau, attaché à la femelle, amorce et conclut la traite manuelle (production laitière moyenne globale de $2000 \mathrm{~kg}$ par lactation). La suppression progressive de cette dernière, l'extension du croisement avec des taureaux de race à viande (charolais surtout) impliquent que soit précisé l'intérèt de ce croisement suivant que les élevages pratiquent ou non la traite et dans le deuxième cas suivant les potentialités fourragères des montagnes, variables avec la nature géologique (granit, basalte) du sol et avec la pluviosité (comprise entre 500 et $1300 \mathrm{~mm}$ d'eau).

En 1965, sur 158 veaux, on a étudié l'effet du croisement (Aubrac $\times$ charolais vs Aubrac $\times A u b r a c$ ) sur la croissance journalière moyenne des veaux à l'estive (début mai-début octobre) dans le cas des montagnes qui pratiquent encore 2 traites par jour : -39 grammes pour les $\delta \hat{\sigma}$ $(\mathrm{P}>0,05)$ et -10 grammes pour les 우 $(\mathrm{P}<0,05)$ et dans le cas de celles où on a abandonné complètement la traite : +91 grammes pour les ${ }^{*} \sigma^{*}(\mathrm{P}<0,01)$ et +112 grammes pour les $\subsetneq$ f $(\mathrm{P}$ $<0,01)$. Ces résultats traduisent une interaction hautement significative entre l'effet du croisement et celui du mode d'exploitation des femelles. En 1966, on a analysé l'effet du croisement sur 
les " montagnes " ne pratiquant plus la traite à cette époque : 90 p. 100 du total environ $(\mathrm{N}=$ 2 132), en fonction de la qualité de la "montagne " appréciée subjectivement d'après le milieu naturel ou artificiel d'élevage (apport d'aliments concentrés) : 4 classes ont été considérées. Les différences de croissance journalière moyenne (Aubrac $\times$ charolais vs Aubrac $\times$ Aubrac) étaient respectivement dans l'ordre de qualité décroissante des montagnes de $+178 \mathrm{~g},+107 \mathrm{~g},+106 \mathrm{~g}$ et $+24 \mathrm{~g}$ pour les $\delta^{\star} \delta^{*}+121 \mathrm{~g},+60 \mathrm{~g},+54 \mathrm{~g}$ et $-38 \mathrm{~g}$ pour les $q$ 우. Ces résultats indiquent encore une interaction croisement $x$ milieu d'élevage dans les montagnes où le veau consomme la totalité du lait de sa mère. Cette même année, on a pu en outre comparer sur des montagnes de bonne qualité la croissance de veaux Aubrac $\times$ Aubrac, Aubrac $\times$ charolais, croisés de retour sur charolais et charolais $\times$ charolais soit des génotypes possédant $0,50,75$ et 100 p. 100 de sang charolais. Les valeurs moyennes de croissance journalière étaient respectivement pour ces 4 catégories de $995 \mathrm{~g}, 1013 \mathrm{~g}, 1009 \mathrm{~g}$, et $902 \mathrm{~g}$ chez les 60 ; de $778 \mathrm{~g}, 767 \mathrm{~g}, 806 \mathrm{~g}, 746 \mathrm{~g}$ chez les 우.

Tous ces résultats traduisent des différences génétiques d'adaptation au milieu (maternel pour le veau, physique pour le couple mère-veau) qu'il convient d'analyser. Ils conduisent à comparer l'efficacité économique de diverses stratégies d'exploitation des croisements entre le cheptel autochtone et les mâles de races à viande, à spécialiser enfin l'élevage dans chaque zone en fonction du génotype le mieux adapté aux caractéristiques de la zone. On a pu observer d'ores et déjà sur le terrain des directions privilégiées d'extension du croisement, directions qui sont fonction des potentialités du milieu.

Sous notre direction, cette recherche a fait l'objet d'un document de fin d'éludes de l'École supérieure d'Agriculture de Purpan par A. Gauthier (1967, ronéoté). Elle s'intègre par ailleurs dans le cadre des travaux de la R.C.P. Aubrac (Recherche coopérative sur Programme) organisés par le C.N.R.S.

\title{
UTILISATION DE TAUREAUX CHAROLAIS, LIMOUSINS ET PIÉMONTAIS POUR LE CROISEMENT INDUSTRIEL EN SARDAIGNE
}

\author{
S. CASU. - Istituto Zootecnico e Caseario per la Sardegna, Sassari.
}

Durant 3 années (1964-65-66), 150 vaches de races sardo-modicana, bruno-sarda et métisse ont été accouplées à 4 taureaux charolais, 2 limousins et 4 piémontais pour la production de veaux de 15 mois ("vitellone "). Après un sevrage à 6 mois, les produits croisés contrôlés ( 85 charolais, 89 limousins et 74 piémontais) ont reçu une ration à base de foin et d'aliment concentré jusqu'à l'abattage. Par une analyse de moindres carrés, on a montré la supériorité pondérale des mâles à tout âge par rapport aux femelles : $1,600 \mathrm{~kg}$ à la naissance, $8,270 \mathrm{~kg}$ au sevrage, $41,100 \mathrm{~kg}$ à 12 mois, $81,700 \mathrm{~kg}$ à l'abattage, $52,800 \mathrm{~kg}$ en carcasse (avec $3,80 \%$ et $0,54 \%$ de gras et d'os en moins). L'année de naissance a une influence significative sur le poids à tous les âges et sur le poids et la qualité des carcasses. Les produits des vaches bruno-sarda et sardo-modicana ont une meilleure vitesse de croissance et des carcasses plus lourdes que les produits des vaches métisses. La race des taureaux n'a pas d'influence sur le poids au sevrage, mais à l'abattage les veaux croisés charolais sont plus lourds de 20 kilogrammes que les autres. La différence des poids de carcasses n'est toutefois que de 10 kilogrammes : les croisés piémontais ont le meilleur rendement à l'abattage, leur carcasse étant de meilleure qualité (gras et os moins importants, pourcentage plus élevé de morceaux nobles), supériorité due en partie au caractère culard des taureaux piémontais utilisés. Les taureaux charolais et piémontais en croisement industriel avec des vaches locales donnent donc des carcasses de meilleure valeur bouchère (poids et qualité) que les taureaux limousins et se révèlent, par conséquent, les plus intéressants pour la production de "vitellone " dans les conditions moyennes d'élevage de la Sardaigne. 